\title{
Article \\ Palliation of Four-Wave Mixing in Optical Fibers Using Improved DSP Receiver
}

\author{
Fazal Muhammad ${ }^{1}$ (D), Farman Ali ${ }^{2}$, Ghulam Abbas ${ }^{3}{ }^{(D)}$, Ziaul Haq Abbas ${ }^{4}$, Shahab Haider ${ }^{5}$, \\ Muhammad Bilal ${ }^{6, *(D)}$, Md. Jalil Piran ${ }^{7}$ and Doug Young Suh ${ }^{8, *}$
}

1 Department of Electrical Engineering, University of Engineering and Technology, Mardan 23200, Pakistan; fazal.muhammad@uetmardan.edu.pk

2 Department of Electrical Engineering, Qurtuba University of Science and IT, D. I. Khan 29050, Pakistan; drfarmanali.optics@qurtuba.edu.pk

3 Faculty of Computer Science and Engineering, GIK Institute of Engineering Sciences and Technology, Topi 23640, Pakistan; abbasg@giki.edu.pk

4 Faculty of Electrical Engineering, GIK Institute of Engineering Sciences and Technology, Topi 23640, Pakistan; ziaul.h.abbas@giki.edu.pk

5 Department of Computer Science, City University of Science and Information Technology, Peshawar 25000, Pakistan; dr.shahab39@gmail.com

6 Department of Computer Engineering, Hankuk University of Foreign Studies, Yongin-si 17035, Korea

7 Department of Computer Science and Engineering, Sejeong University, Seoul 05006, Korea; piran@sejong.ac.kr

8 Department of Electronics and Information Convergence Engineering, Kyung Hee University, Seoul 17104, Korea

* Correspondence: m.bilal@ieee.org (M.B.); suh@khu.ac.kr (D.Y.S.)

Citation: Muhammad, F.; Ali, F.; Abbas, G.; Abbas, Z.H.; Haider, S.; Bilal, M.; Piran, M.J.; Suh, D.Y.

Palliation of Four-Wave Mixing in Optical Fibers Using Improved DSP Receiver. Electronics 2021, 10, 611. https: / / doi.org/10.3390/

electronics10050611

Academic Editor: Davide De Caro

Received: 28 January 2021

Accepted: 28 February 2021

Published: 5 March 2021

Publisher's Note: MDPI stays neutral with regard to jurisdictional claims in published maps and institutional affiliations.

Copyright: (c) 2021 by the authors. Licensee MDPI, Basel, Switzerland. This article is an open access article distributed under the terms and conditions of the Creative Commons Attribution (CC BY) license (https:// creativecommons.org/licenses/by/ $4.0 /)$.

\begin{abstract}
A long haul optical communication system (LHOCS) is one of the key resources to fulfill the higher capacity requirements in future communication networks. To launch LHOCS, the system mainly faces high order nonlinear effects. The four-wave mixing (FWM) is one of the major nonlinear effects, which limits the transmission distance. Therefore, in this paper, an advanced duo-binary (DB) modulation scheme-based system is evaluated by employing an improved digital signal processing (IDSP) approach at the receiver side to suppress the FWM effect. In addition, an analytical analysis is also performed for the proposed system. To observe the difference between the IDSP and conventional digital signal processing (DSP), the various performance metrics such as bit error rate (BER), Q-factor, and optical signal-to-noise ratio (OSNR) parameters are evaluated. Variable channel spacing along with polarization mode dispersion (PMD) are analyzed at several ranges of input powers and fiber lengths. The analytical and simulation-based calculations exhibit the effectiveness of the proposed model and hence, FWM effect are compensated to achieve $500 \mathrm{~km}$ optical fiber propagation range with a BER below $10^{-6}$.
\end{abstract}

Keywords: improved digital signal processing; four-wave mixing nonlinear effect; duo-binary modulation; long haul optical communication system; variable channel spacing

\section{Introduction}

The Internet applications and services have become a pervasive presence in our daily lives. Due to the development of handhold devices for various applications, technological advancements in telecommunication industry have become a prime need [1-4]. For last mile technologies, the end-to-end system requires an ultra-high capacity network to support millions of devices [5,6]. Likewise, high bandwidth throughput are needed for the security and monitoring structures of mobile network base stations (BSs). In the 1980s, the deployment of optical fibers was started for long-haul systems and it was assumed that they had unlimited transmission bandwidth at least for any foreseeable data rate requirements $[7,8]$. Furthermore, telecommunication services such as broadband, cable 
transmission, and other interactive multimedia services need optical fiber as a transmission medium owing to its excessive capacity and huge bandwidth [9-11]. As the need arises to send huge capacity data across long transmission range, the optical communication industry starts to use wavelength division multiplexing (WDM) that is used to ship diverse signals through a fiber [12]. With the help of employing this technology in long haul optical communication system (LHOCS), the transmission capacity can be increased several folds. However, on the other side, this WDM technology induces extra unwanted frequencies, which badly interrupt the overall performance of LHOCS. The noises generated due to these unwanted induced frequencies are named as nonlinear effects (NLEs) $[13,14]$. These NLEs are classified into two types including scattering related NLEs $[15,16]$ and refractive index related NLEs $[17,18]$. The scattering related NLEs are known as Stimulated brillion scattering (SBS) [19] and stimulated Raman scattering (SRS) [20]. On the other hand, the refractive index related NLEs include four wave mixing (FWM) [21], self-phase modulation (SPM) [22] and cross phase modulation (XPM) [23]. These aforementioned types have severe effects on performance of LHOCS. However, FWM has a strong impact on LHOCS compare to other NLEs because of WDM technology and less channel spacing [24]. In WDM systems, several optical signals are transmitted, having less frequency spacing among them, over a single mode fiber (SMF). Hence, as a result, the transmitting optical signals are mixed with each others and causes the generation of extra unwanted signals. This phenomena is known as FWM. This effect becomes adverse as the number of user increases in LHOCS. Therefore, existing system of optical communication system (OCS) is not capable of countering FWM effectively in long haul transmission. The FWM nonlinear effects are the most common limiting factors in long haul transmission, the accumulated chromatic dispersion (CD) and polarization mode dispersion (PMD) have also been reported in several references to cause adverse effects in optical fiber transmission. CD is a severe problem in WDM systems, as different wavelengths travel through the same fiber. The long-haul transmission give rise to the accumulated CD [25] in a wavelengthdependent setup, which can be compensated by using slope compensation but it causes an increase in FWM nonlinear distortions [26]. As compared to linear accumulation of $\mathrm{CD}$, the PMD accumulates stochastically along the fiber length and causes distortion and reduction in peak power for long-haul fiber-optic transmission with high data rates [27]. Therefore, an improved OCS framework is a much needed requirement, which has the capability to overcome the effects of CD, PDM, and FWM.

\subsection{Related Work}

To ensure transmission integrity, a large volume of literature exists where different schemes were presented for mitigating the FWM effect. Some advanced contributions and their limitations are presented as follow: fiber optical parametric amplification (FOPA) [28], electronic absorption modulation process with different optical filters such as infinite impulse response (IIR), Butterworth, and Bessel [29] are the advance procedure for controlling the impact of FWM. In [30] fiber bragg grating (FBG) technique is used for long haul networks to reduce FWM effect and a method for minimizing FWM impact using channel spacing technique was reported in [31]. Digital signal processing (DSP) techniques for compensating inter-and intra-channel fiber non-linearities have been investigated in [32] by manipulating input parameters such as launch power and fiber length. Self phase modulation (SPM) non-linear issue has been investigated in [33] where conversion between different formats, such as 8-quadrature amplitude modulation (8-QAM) to quadrature phase shift keying (QPSK) and to on-off-keying (OOK) has been performed through optical methods to adapt the channel conditions. Different values of optical signal-to-noise ratio (OSNR), optical power, and chromatic dispersion were used to conduct analysis on the performance of the proposed system; however, the format conversion to a lower order results in decrease of data rates. In [34], the authors discuss inter-model nonlinear issues in few-mode fiber using 16-QAM up to $36 \mathrm{~km}$ transmission and report mitigation of the XPM effect. High cost components have been used in [35] such as indium phosphate and silicon 
photonics to achieve high bandwidth in the presence of fiber non-linearities. The voltra assisted method was explained in [36] in which the authors overcome the limitations of optical phase conjugation (OPC) technique. All above mentioned techniques have higher complexity and are not cost efficient. Hence, FWM effect still need to be investigated in depth for LHOCSs. Moreover, modern LHOCSs require multiplexing of several channels for which different parameters of a WDM-based system need to be analyzed. Therefore, we present a detailed analysis of FWM by varying input power, fiber length, channel spacing, and multichannel. To evaluate the executions of the presented LHOCS, an in depth investigation is carried out theoretically and experimentally.

\subsection{Our Contributions}

As discussed above, the traditional OCS cannot meet the challenges such as data rate, capacity, privacy and long distance due to the FWM effect. Therefore, to support user demands, a new set-up is required, where FWM is managed and high data rate, long range transmission, low latency and less complexity goals are provided to the users. Henceforth, to address these issues an analytical and experimental mechanism is developed in this work to maximize the system performance and handle FWM effect. The major handouts of this model are mentioned as follows.

(A) We present a new model coined as LHOCS, where the FWM are compensated and high capacity information is communicated over a $500 \mathrm{~km}$ distance.

(B) An analytical model is presented with an improved digital signal processing (IDSP) receiver to mitigate FWM effect and support 8, 16, and 32 WDM channels.

(C) We also show that transmission of 100 Gbps data rate over $32 \mathrm{WDM}$ channels is achieved for $500 \mathrm{~km}$ length of optical fiber.

(D) The duo-binary (DB) advanced modulation scheme is applied to narrow the pulse spectrum, which leads to manage FWM successfully over longer distances.

(E) Through extensive simulation we demonstrate that our proposed technique work better compare to current optical communication system in terms of BER, length and capacity.

The rest of paper is organized as follows. The network layout is studied in Section 2. Section 3 presents the theoretical background. The results and discussion is analyzed in Section 4 . Finally, Section 5 concludes the paper.

\section{System Model}

The objective of the proposed work is to design a model for the compensation of FWM effect. The fundamental concept and network architecture of LHOCS is explained in this section. A 32 channel-based proposed framework considered in this work is shown in Figure 1. The transmitter of the proposed setup is using 32 continuous wave (CW) laser array having 12.5, 25, and $50 \mathrm{GHz}$ channel spacing is shown in Figure 1. The spectral width of the optical signal at transmitter side is narrow down by employing advanced DB modulation scheme. Moreover, by implementing DB, the chromatic dispersion effect is compensated due to its three level signal (electrical form) property [37]. Modulated DB optical pulses are achieved by using a mach-zehnder modulator (MZM). The output signals of filtered pseudo random bit sequence (PRBS) generator are used to drive MZM. These filtered signals are generated by DB precoder after introducing one bit delayed line in electrical domain in order to set the levels of correlated signals for DB modulation. Moreover, MZM driver ensures the availability of $2 V_{p i}$ full swing and controls the bias voltage for DB modulation. Then, the modulated signals are fed to a WDM multiplexer, having $3 \mathrm{~dB}$ insertion loss, for transmitting over SMF. SMF of length $500 \mathrm{~km}$ is used for data transmission. Since the attenuation of SMF is $0.2 \mathrm{~dB} / \mathrm{km}$, therefore, to manage such losses erbium doped fiber amplifiers (EDFAs) are used after each $100 \mathrm{~km}$ fiber of length. At the receiver side, after demultiplexing, the optical to electrical conversion is performed using photo detector. Thus, DB modulated signals are processed as a simple NRZ demodulation process with only two decision levels. The received electrical signal is then passed 
through an electrical filter and RF amplifier to reduce the amplified spontaneous emission (ASE) issues. In addition, to counter the effect of FWM, IDSP is applied, which is described in Section 2.2. The list of different parameters used in the proposed model are summarized in Table 1, where most of the elements are kept constant to simulate the system presented in Figure 1. On the other, side parameters such as channel spacing, number of channels, linear and nonlinear dispersion, transmission range, nonlinear effective area, nonlinear refractive index, dispersion slope and input power are varied within a limited range for analyzing the performance against FWM. Likewise, values of the parameters used in this work are listed in Table 1.

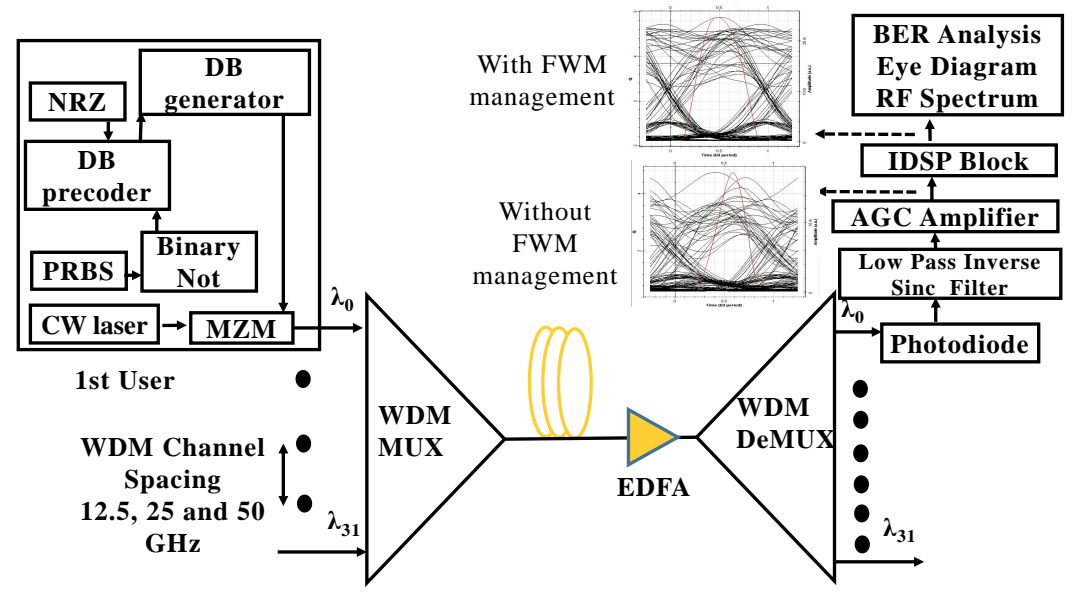

Figure 1. Main setup of LHOCS for 32 channels, 100 Gbps speed and $500 \mathrm{~km}$ transmission range.

Table 1. Simulation Parameters.

\begin{tabular}{cc}
\hline Description & Magnitude \\
\hline Line width & $10 \mathrm{MHz}$ \\
Initial phase & $0 \mathrm{deg}$ \\
Launch power & $-6-6 \mathrm{dBm}$ \\
output power & -27 to $-16 \mathrm{dBm}$ \\
Spacing among transmitted multichannel & 50 to $200 \mathrm{GHz}$ \\
Transmission path & $500 \mathrm{~km}$ \\
Phase modulation dispersion & $0.6 \mathrm{ps} / \mathrm{km}^{2}$ \\
Multichannel range & 32 \\
Noise bandwidth & $640 \mathrm{GHz}$ \\
Nonlinear dispersion & $-3 \mathrm{ps}^{3} / \mathrm{km}$ \\
EDFA amplifier & Gain controller of $20 \mathrm{~dB}$ \\
\hline
\end{tabular}

\subsection{FWM}

The current OCS operates at few milliwatt power, bit rate up to $5 \mathrm{Gbps}$ and bandwidth around $5 \mathrm{GHz}$. Such type of systems are considered to be linear systems [38]. However, data rate greater than $10 \mathrm{Gbps}$, bandwidth above $10 \mathrm{GHz}$ and high transmitting power leads towards the introduction of NLEs in the fiber [39]. For WDM systems, the NLEs become more severe [40]. The main effect out of these NLEs is FWM. FWM is the intermodulation phenomenon, whereby interactions among three wavelengths produce the fourth wavelength. In a WDM system, it induces phase shift within a channel and new frequencies are generated as illustrated in Figure 2. The $\vartheta_{k}, \vartheta_{l}$ and $\vartheta_{m}$ are the original transmitted wavelengths while others are unwanted generated wavelengths due to FWM. The FWM effect increases while channel spacing decreases in LHOCS. Thus, the impact of FWM becomes more significant for closely spaced channels' based system even at low data rates. To eliminate the effect of FWM in LHOCS, we investigate IDSP receiver management and polarization mode approach in this paper. 


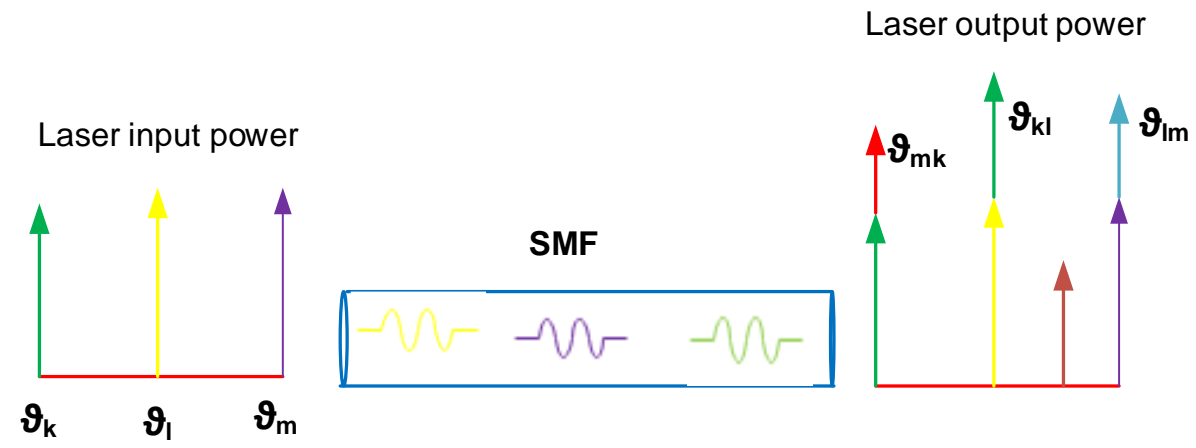

Figure 2. Induction of unwanted frequencies due to FWM.

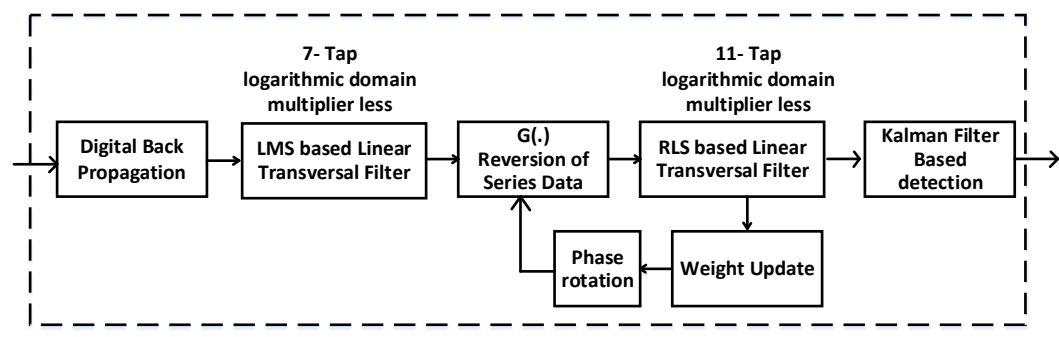

Figure 3. IDSP receiver internal framework.

\subsection{Improved DSP}

It is concluded from the proposed model that FWM effects can be mitigated by use of advance duo-binary modulation scheme for a WDM-based LHOCS and further improvement in the performance can be achieved with a IDSP receiver rather than using conventional dispersion compensation fiber (CDCF) for dispersion compensation. The IDSP based receiver is presented in Figure 3, which describes that two cascaded least mean square (LMS) and recursive lease square (RLS) transversal filtered are installed, including 7 and 11 taps, respectively. This integration approach of LMS and RLS present excellent performance and fidelity against the error signals. Moreover, LMS and RLS filters offer faster convergence and smaller error, in results the time convergence of the system is enhanced against variable channel impairments. The number of taps are optimized for the proposed system, for the minimum least square error (LSE) and rapid convergence time. Blind adaptive equalization is applied in order to avoid the used of training sequence and complexity of the proposed LHOCS, using constant modulus algorithm (CMA). The effect of FWM nonlinearities inside LHOCS are multiplicative in nature, thus, for detecting the received bits an extended Kalman filter [41] is installed instead of matched filter. It uses a likelihood threshold (LTR) method, aiming to adjust receiver orientation, and updates the threshold after each iteration.

\subsection{Polarization Mode Dispersion}

The phase of transmitted signals are distorted owing to high capacity and long cover path. The transmitted signals are separated into multiple mode as arrived at the receiver side with a slightly different time as described in Figure 4. In results the phase is changed of the received signal and the pulse gets broader. At higher distance and speed, the impact of phase change and pulse broadening are increased further. 

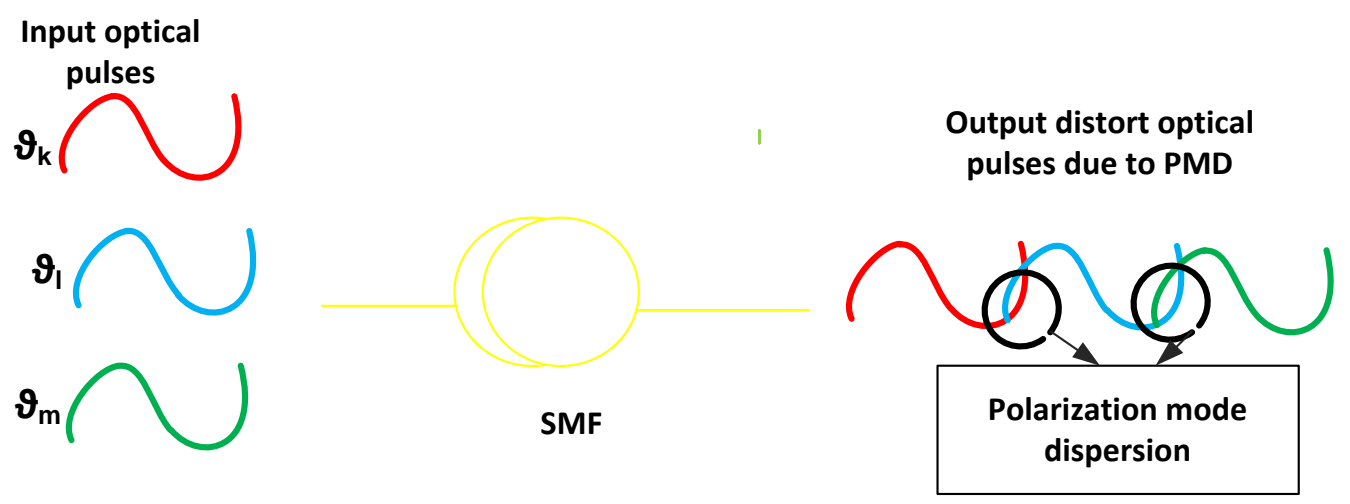

Figure 4. Distortion of propagated optical signal due to phase mode dispersion.

Therefore, it is shown in Figure 4 that PMD is the key source of transmitted signal degradation and positive BER and FWM effect. To overcome the PMD issues a fruitful management technique is necessary for achieving acceptable range of BER.

\section{Analytical Modeling}

The proposed system aims to maintain system fidelity for long distance transmission at high data rates. This section presents a detailed analytical model for analyzing FWM. The parameters that affect the severity of FWM are the SMF cross sectional area, magnitude of laser light, multi channel WDM system with high data rate speed. Inside SMF the inharmonic movements of photons are induced by reason of these mentioned factors [42], and gives nonlinear induced polarization $\Omega_{P}$ [43], which is given as:

$$
\Omega_{P}=\varepsilon_{0} \kappa^{\prime} E+\varepsilon_{0} \kappa^{\prime \prime} E^{2}+\varepsilon_{0} \kappa^{\prime \prime \prime} E^{3},
$$

where $\varepsilon_{0}$ is free space permittivity, $E$ is electrical field intensity and $\kappa^{\prime}, \kappa^{\prime \prime}$ and $\kappa^{\prime \prime \prime}$ are first, second and third order susceptibility, respectively.

The effects of FWM for optical medium are originated from the electric dipole polarization induction, which is mainly due to the $\kappa^{\prime}$ while the $\kappa^{\prime \prime}$ does not contribute for silica based propagation mediums [44] and $\kappa^{\prime \prime \prime}$ produces very low order nonlinear effects. Thus, by neglecting $\kappa^{\prime \prime}[45]$ the induced polarization from (1) becomes:

$$
\Omega_{P}=\varepsilon_{0} \kappa^{\prime} E \cos (\Phi t-z x)+\varepsilon_{0} \mathcal{K}^{\prime \prime \prime} E^{3} \cos ^{3}(\Phi t-z x) .
$$

By applying the trigonometric property of expansion on (1) and neglecting constant terms and $3 \Phi$ terms, we get:

$$
\Omega_{P}=\varepsilon_{0} \kappa^{\prime} E \cos (\Phi t-z x)+\frac{3}{4} \varepsilon_{0} \kappa^{\prime \prime \prime} E^{3} \cos ^{3}(\Phi t-z x) .
$$

In (3), both linear and nonlinear parameters are included.

For the phase wave explored in (2), the intensity $\iota$ of pulse [46] is defined as

$$
\iota=\frac{1}{2} c \varepsilon_{0} n_{0} E_{0}^{2}
$$

where $c$ denotes the light velocity, and linear refractive index is represented by $n_{0}$ at low electrical fields [47]. Thus, (3) simplifies to

$$
\Omega_{P}=\varepsilon_{0} \kappa^{\prime} E \cos (\Phi t-z x)+\frac{3}{2} \frac{\kappa^{\prime \prime \prime}}{c \varepsilon_{0} n_{0}} \iota E \cos (\Phi t-z x) .
$$


The effective susceptibility [48], $\kappa_{e}$, of the optical medium is calculated as

$$
\kappa_{e}=\kappa^{\prime}+\frac{3}{2} \frac{\kappa^{\prime \prime \prime}}{c \varepsilon_{0} n_{0}} l,
$$

and the effective refractive index, $n_{e}$, is measured as

$$
n_{e}=n_{0}+n_{r} l,
$$

where $n_{r}$ represents nonlinear refractive index, which is given as

$$
n_{r}=\frac{3}{4} \frac{\kappa^{\prime \prime \prime}}{c \varepsilon_{0} n_{0}^{2}}
$$

In addition, FWM increases with the increase in length of fiber. As length of fiber increases, the interaction of a signal with the fiber and other traveling signals increases, which results in enhancing the effect of FWM [49]. This optical power, in terms of length of fiber, $L$, and attenuation, $\alpha$, is written as

$$
\rho_{r}=\rho_{i} \exp -\alpha L,
$$

where $\rho_{i}$ and $\rho_{r}$ are input and output powers, respectively. For length, $L$, the effective length, $L_{e}$, of the fiber is given as

$$
\rho_{i} L_{e}=\int_{L=0}^{L=w} \rho_{r}(L) d L .
$$

From (9) and (10), $L_{e}$ can be written as

$$
L_{e}=\frac{1-\exp (-\alpha L)}{\alpha} .
$$

The effective length after placing optical amplifiers with $L$ and amplifier spaced distance $z[50]$, is defined as

$$
L_{e}=\frac{1-\exp \left(-\alpha L^{2}\right)}{\alpha} \frac{L}{z} .
$$

Cross sectional area, $\xi_{e f f}$, of fiber core has inverse relation to $\iota$ [51] and can be estimated as

$$
\xi_{e f f}=\frac{\int_{m} \int_{b} m d m d b}{\int_{m} \int_{b} m d m d b \iota(m, b)}
$$

where $m$ and $b$ are the polar coordinates. Due to the SPM, different parts of the optical signal undergo different phase shifts, which results in frequency chirping. Broadening of spectrum is the initial impact of SPM, which further increases for high launch power. Hence, based on wavelength, $\lambda, L$, and $E$, the phase, $\omega$, is defined as

$$
\omega=\frac{2 \pi}{\lambda} n L,
$$

where $n$ represents refractive index. In terms of linear and nonlinear phenomenon, (14) is written as

$$
\omega=\frac{2 \pi}{\lambda}\left(n_{0}+n_{r} \iota L_{e}\right)
$$


The first portion of the above equation is called linear phase $\omega_{0}$, and the second portion is known as nonlinear phase shift $\omega_{r}$. For time dependent phase angle, the modulated optical carrier frequency, $\vartheta_{c}$, based on frequency spectrum, $\vartheta_{0}$, is given as

$$
\vartheta_{c}=\vartheta_{0}+\frac{d \omega}{d t} .
$$

Furthermore, the nonlinear phase shift, $\omega_{r}$, can be written as

$$
\omega_{r}=\int_{0}^{L}\left(H_{e}-H_{0}\right) d m .
$$

When multiple channels are transmitted, then the phase shift of the signal is generated due to co-channels. Therefore,

$$
\omega_{r}=H_{e} L_{e}\left(\rho_{v 0}+2 \rho_{v 1}\right),
$$

where $\rho_{v 0}$ and $\rho_{v 1}$ are the powers of two co-channels.

From (1) to (3), it is described that induced polarization composes of both linear and FWM terms. The FWM nonlinear impact is caused by the third order susceptibility. In this process, photons of transmitted signals annihilate and result in production of new photons at various frequencies. In contrast to SPM and XPM, FWM is independent of bit rate and dependent on channel spacing and fiber dispersion. To calculate the effect of FWM, the induced polarization is written as:

$$
\begin{aligned}
\Omega_{P}= & \frac{3}{4} \varepsilon_{0} \kappa^{\prime \prime \prime} \sum_{a=1}^{i} E_{a}^{2}+\frac{3}{4} \varepsilon_{0} \kappa^{\prime \prime \prime} \sum_{a=1}^{i} \sum_{a>s} \sum_{s>l} E_{a} E_{s} E_{l} \\
& \cos \left(2 \vartheta_{i i}+\vartheta_{j j}+\vartheta_{k k}\right) t-\left(H_{i i}+H_{j j}+H_{k k}\right) z \\
& +\cos \left(\vartheta_{i i}+\vartheta_{j j}+\vartheta_{k k}\right) t-\left(H_{i i}+H_{j j}+H_{k k}\right) z \\
& +\cos \left(\vartheta_{i i}-\vartheta_{j j}+\vartheta_{k k}\right) t-\left(H_{i i}-H_{j j}+H_{k k}\right) z \\
& +\cos \left(\vartheta_{i i}-\vartheta_{j j}-\vartheta_{k k}\right) t-\left(H_{i i}-H_{j j}-H_{k k}\right) z .
\end{aligned}
$$

The primary portion in the above equation represents SPM and XPM, while the secondary part denotes FWM. It explains that FWM occurs due to mixing of different frequencies. As FWM are the main causes of power penalty and system performance degradation, the proposed system mitigates their effects by employing NRZ, DB modulation, large effective area and uneven channel spacing limited by controlling nonlinear dispersion through a dispersion compensation fiber (DCF).

FWM causes generation of new unwanted mixed frequencies, $f_{\text {new }}$, in WDM optical transmission framework in the range of original frequencies as shown in Figure 3. The unwanted signals also known as beat frequencies can be written as

$$
f_{\text {new }}=f_{l} \pm f_{m} \pm f_{n},
$$

where $f_{l}, f_{m}$ and $f_{n}$ are the frequencies of the input signals and $f_{\text {new }}$ is the new generated frequency. (19) and (20) generate new idlers, which interface nearby signals and introduce new frequencies, which can be written as:

$$
\begin{gathered}
f_{i}+f_{j}-f_{k}, \\
f_{j}+f_{k}-f_{i}, \\
f_{j}+f_{k}-f_{i}, \\
f_{i}+f_{k}-f_{j}, \\
f_{j}-f_{i}, \\
f_{i}-f_{j},
\end{gathered}
$$




$$
\begin{gathered}
f_{j}-f_{k} \\
2 f_{k}-f_{j}
\end{gathered}
$$

The power of the FWM effect for continuous spectrum in long range fiber, $P_{\mathrm{FWM}}$, is given as

$$
\begin{aligned}
P_{\mathrm{FWM}}= & 4 \gamma L_{e} P_{i} P_{j} P_{k}\left(N_{s}\right)^{2} C / \lambda^{2} B 2 D \sqrt{(2 / a)^{2}} \\
& +\frac{2 L^{2}(1-C R)^{2}\left(\left(N_{s}\right)^{2}-1\right)}{\sum_{w}^{l} \frac{1}{1+\alpha L(1-C R)^{2}}} \times \log \left[N_{c h}^{2} \pi \alpha^{2} D B^{2}\right. \\
& \left.\times \sqrt{\left(\frac{2}{a}\right)^{2}+2 L(1-C R)^{2}\left(N_{s}^{2}-1\right)}\right] .
\end{aligned}
$$

where $w=\lambda^{2} B^{2} D L(1-C R)\left(N_{c h}\right)^{2} / 16 c, l=\lambda^{2} \psi_{B}^{2} D L(1-C R)\left(N_{c h}\right)^{2} / 2 c, N_{c h}$ is the number of channels used in WDM-based optical fiber system, $\gamma$ represents nonlinear coefficient and is given as

$$
\gamma=\frac{\left(2 \mathrm{n}_{2}\right)}{\lambda \omega^{2}}=\frac{\left(6.28 \mathrm{n}_{2}\right)}{\left(\lambda_{e f f} \times A_{e f f}\right)}
$$

where $D$ is used for dispersion of signal, $\alpha$ explores the attenuation of optical fiber, $L_{e}$ is the effective fiber length, $L$, is the span of the installed fiber, number of span is denoted by $N_{s}$, continuous frequency range is described by $C R, c$ is speed of light, $\lambda_{\text {eff }}$ means effective wavelength and $\psi_{B}$ depicts the bandwidth. The $L_{e}$ is given by

$$
L_{e}=\left(1-e^{-\alpha L}\right) / \alpha,
$$

$\psi_{B}$ of the WDM system with channel spacing is defined by

$$
\psi_{B}=(N-1) \Delta f,
$$

$P_{\text {FWM }}$ depends on channel spacing $\delta f_{c}$ and can be written as

$$
\delta f_{c}=n \times \Delta f,
$$

Currently, the error vector machine (EVM) is extensively used for transmission network as a performance metric. EVM basically a root mean square (RMS) value to distinguish the transmitted and received symbols can be written as

$$
E V M_{R M S}=\frac{I}{M} \sum_{n=1}^{M}\left|R_{x, n}-T_{x, n}\right|^{2} / \rho_{a}
$$

where $M$ is the estimated symbols $R_{x, n}$ and $T_{x, n}$ are received and transmitted directional signals and $\rho_{a}$ is the average power to be transmitted for DB modulation. Thus, in this model the BER is estimated from EVM is explained as

$$
B E R=\frac{\left(1-L^{-1 / 2}\right)}{\frac{1}{2} \log _{2} L} \operatorname{erf} \sqrt{\frac{3 / 2}{(L-1) E V M_{R M S}^{2}}},
$$

where $L$ the number of array constellations for DB modulation. The above generalized equations are used to model and investigate the FWM nonlinear parameters in the LHOCS.

\section{Results and Discussion}

As described in the previous sections, OCS is one of the modern modes of telecommunication networks. In order to communicate over a long distance with optical fiber for high 
capacity data transmission, the NLEs such as XPM, SPM, and FWM effects dominate. This work developed the theoretical modeling studied in Section 3 to quantify the balancing of FWM. In addition, the FWM is analyzed for various values of input power, length of fiber, nonlinear effective area, nonlinear refractive index, number of channels, and channels spacing. For the model proposed in Figure 1, the performance of the model is evaluated for different lengths of fiber and BER against 2, 4, and $6 \mathrm{dBm}$ transmitted power as is shown in Figure 5.

The optimum improvement is shown in Figure 6 with managed FWM as compared to without managed FWM. AT $100 \mathrm{~km}$ transmission range, $10^{-1}$ BER is enhanced for managed FWM than without managed FWM. The system performance is analyzed for power penalty and transmission span in terms of $12.5,25$ and $50 \mathrm{GHz}$ channel spacing as described in Figure 6.

The power penalty of $0.5 \mathrm{dBm}$ is observed between operating with and without managed FWM as shown in Figure 6. Furthermore, narrow channel spacing decreases the system performance due to FWM. Figure 7 demonstrates the FWM for 12.5, 25, and $50 \mathrm{GHz}$ channel spacing, $100 \mathrm{Gbps}$ data rate and 32 channels using nonlinear effective area and BER parameters.

The system outcomes in the presence of FWM effect are attained below margin line at $80 \mu \mathrm{m}^{2}$ nonlinear effective area. Moreover, it can be observed from Figure 7 that transmission channels with $50 \mathrm{GHz}$ channel spacing accommodate huge capacity and large propagation cover path due to less FWM as compared to 12.5 and $25 \mathrm{GHz}$ channel spacing. The results between symbol rate and BER are shown in Figure 8 for 12.5, 25, and $50 \mathrm{GHz}$ channel spacing. It also compares the results for 16 and 32 channels at 60 and $100 \mathrm{Gbps}$ data rate. High capacity with low channel spacing $(12.5 \mathrm{GHz})$ transmission produces FWM greater than 25 and $50 \mathrm{GHz}$ channel spacing in LHOCS. Figure 9 includes the proposed model efficiency against FWM in terms of input power and BER for 8, 16, and 32 channel in LHOCS at 25 and $50 \mathrm{GHz}$ channel spacing. This clarifies that $100 \mathrm{Gbps}$ capacity at $25 \mathrm{GHz}$ channel spacing attains BER margin line early as compare to $50 \mathrm{GHz}$ channel spacing based LHOCS.

LHOCS with $500 \mathrm{~km}$ fiber range induces bad outcomes contrasting with $400 \mathrm{~km}$ transmission. The proposed LHOCS functions are investigated in terms of nonlinear dispersion and BER to measure FWM for 400 and $500 \mathrm{~km}$ span of transmission as explored in Figure 10, which shows that the findings of LHOCS decreases with enhancing nonlinear dispersion. Moreover, Figures 11 and 12 compare the performance of presented system in terms of managed FWM using the oscilloscope visualizer and BER analyzer. The attained signals from the proposed model as mentioned in Figures 11a,b and 12a,b, explore that huge amount of noise exist due to without compensation of FWM effect. The results after addressing of FWM effect are clearly different from the uncompensated FWM effect model.

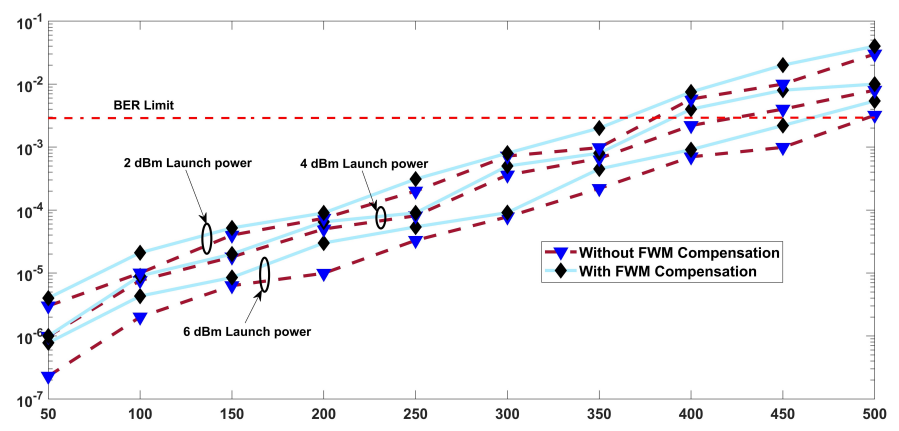

Figure 5. Length of fiber against BER for 2, 4 and $6 \mathrm{dBm}$ input power, comparing operation with and without FWM management techniques. 


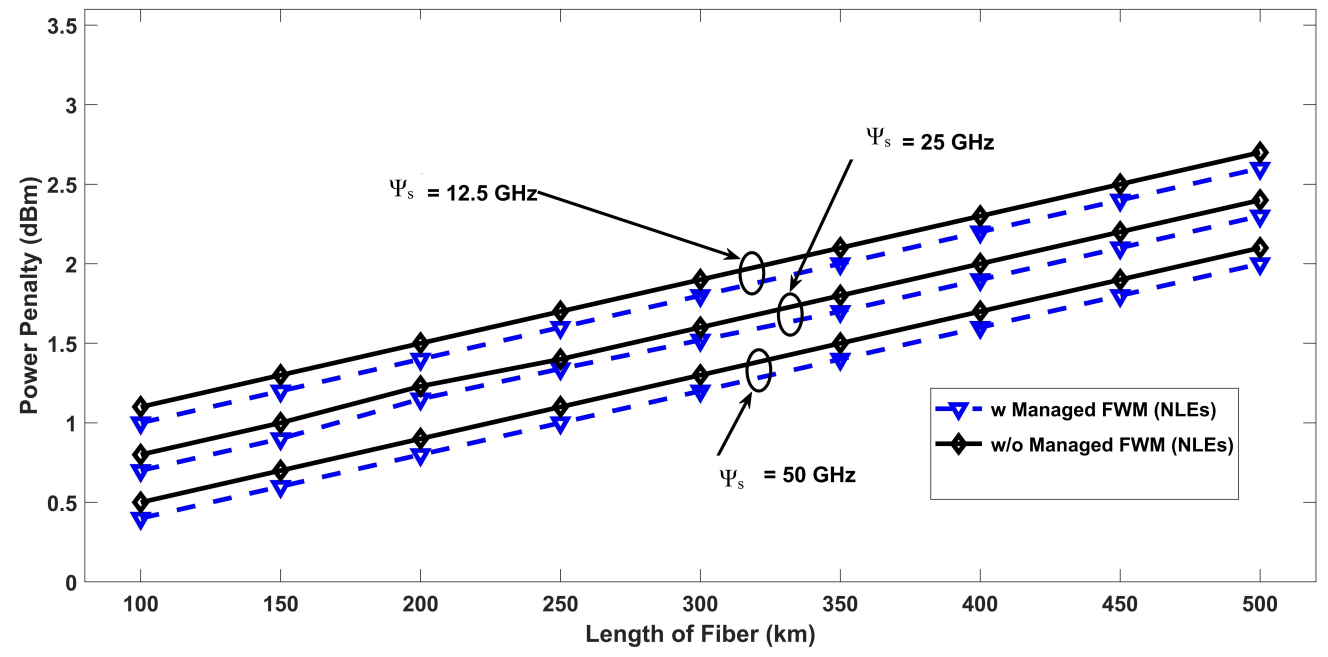

Figure 6. Comparison of power penalty as a function of fiber length for with and without FWM using $12.5,25$, and $50 \mathrm{GHz}$ channel spacing.

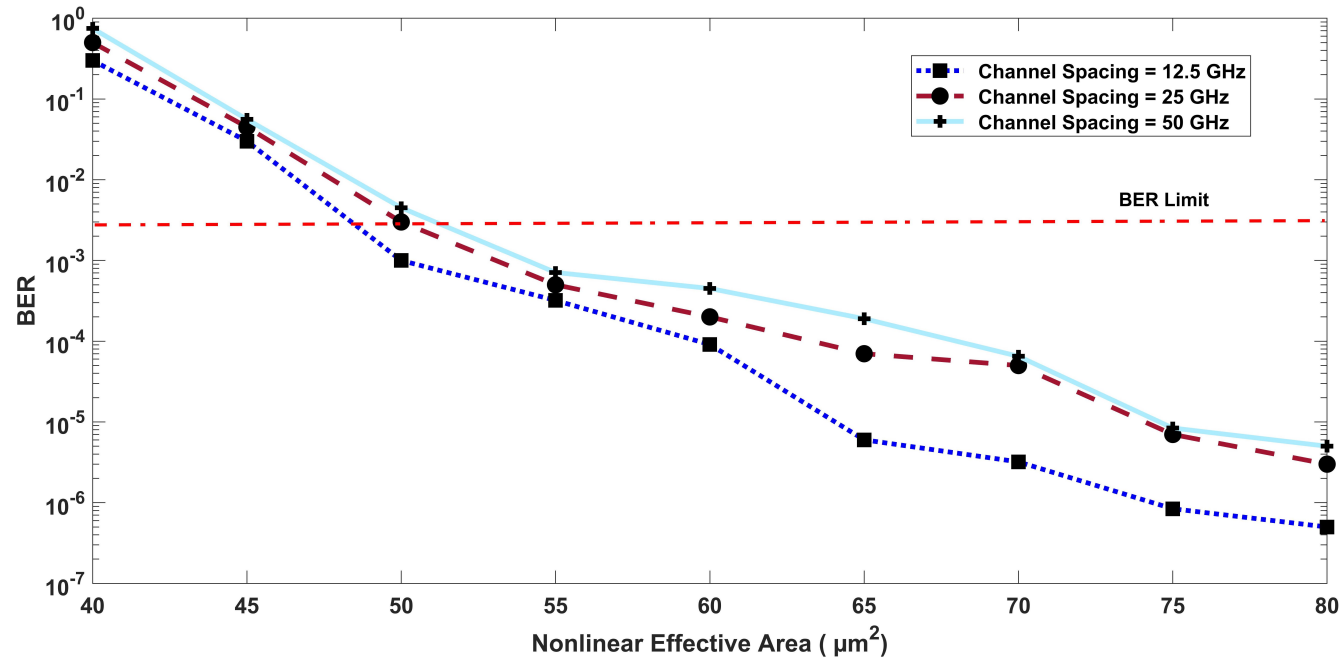

Figure 7. 12.5, 25, and $50 \mathrm{GHz}$ channel spacing comparison applying nonlinear effective and BER calculating parameters for $100 \mathrm{Gbps}$ speed and channel $=32$.

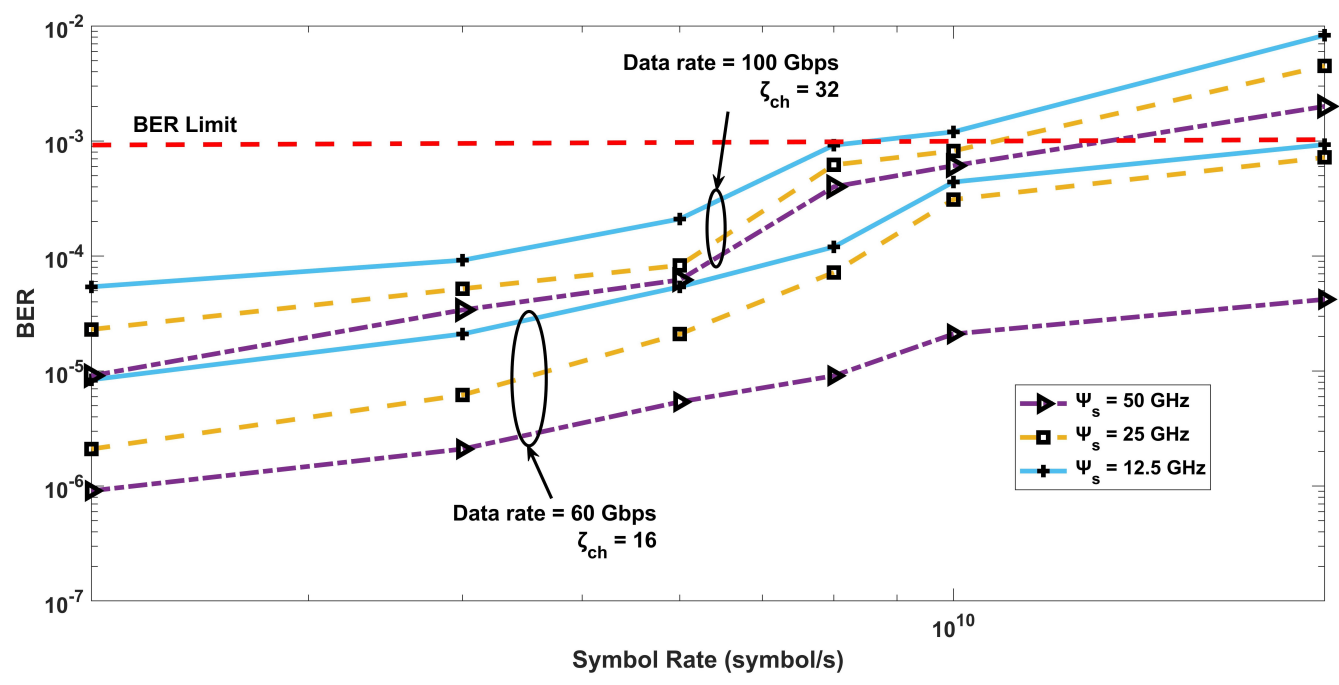

Figure 8. Symbol rate against BER for 12.5, 25, and $50 \mathrm{GHz}$ channel spacing. 


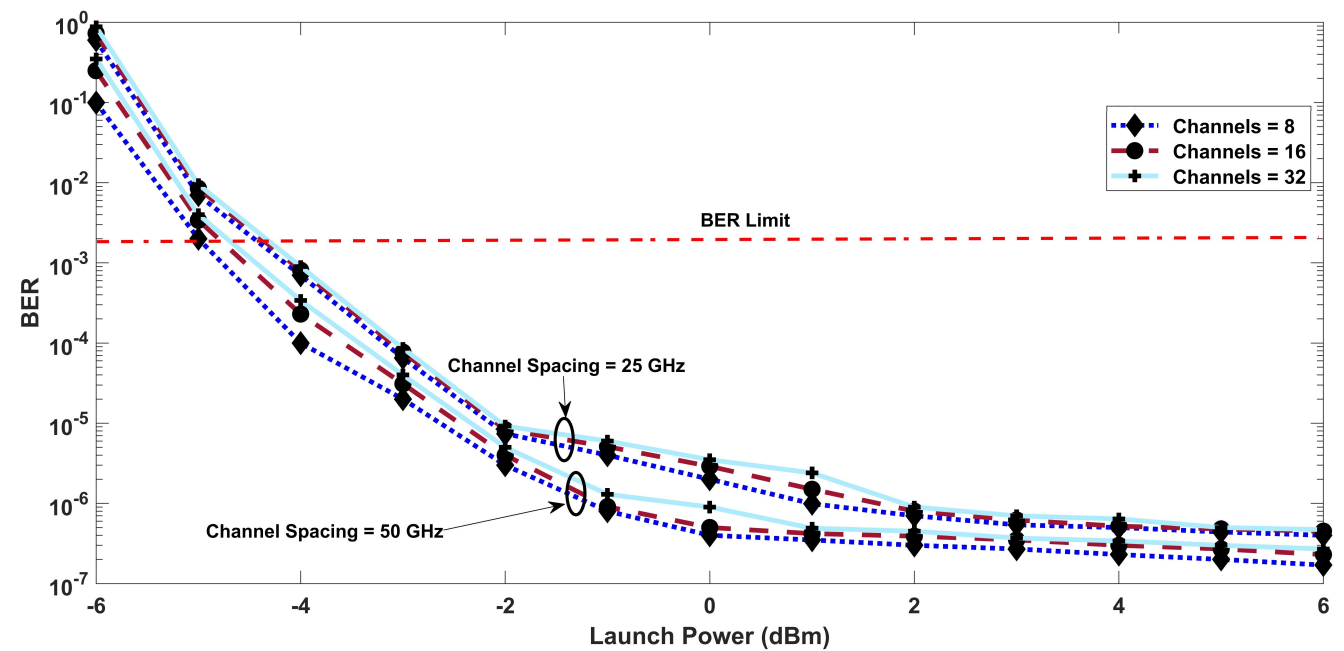

Figure 9. Optical input power vs BER for 8, 16 and 32 channels.

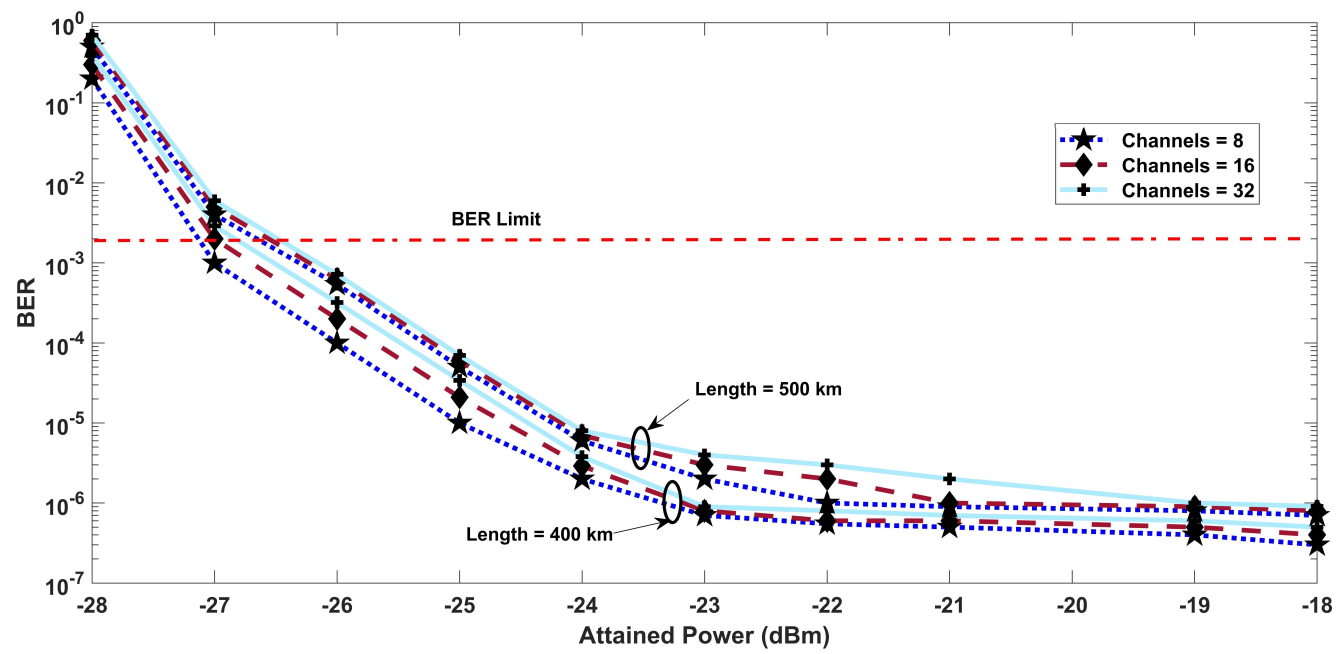

Figure 10. Optical received power against BER for analyzing FWM using 400 and $500 \mathrm{~km}$ fiber length and 8,16 and 32 channels.

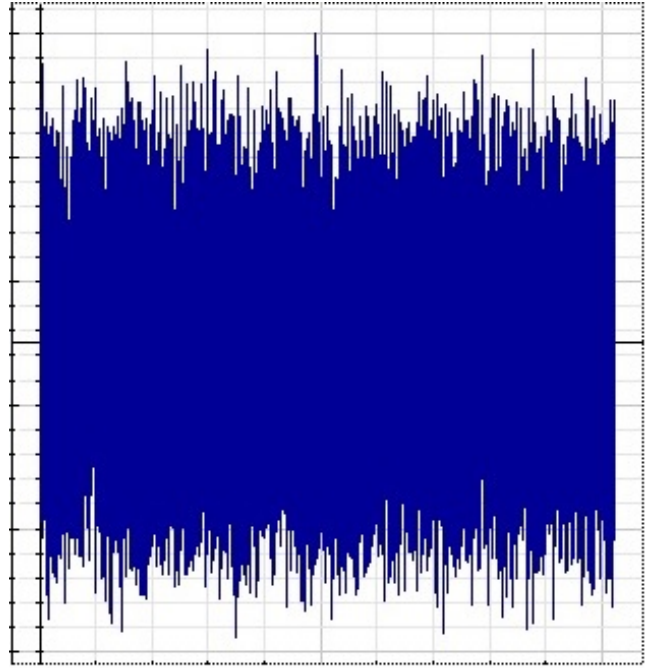

(a)

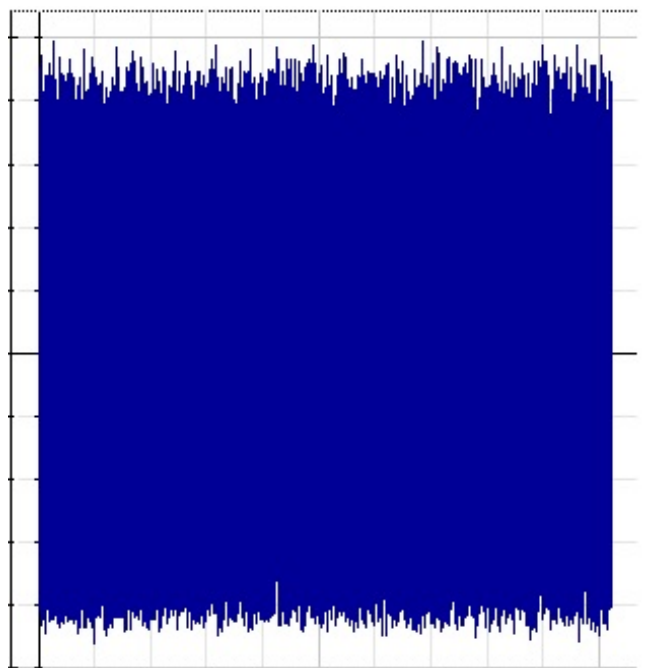

(b)

Figure 11. RF spectrum analyzer. (a) Without addressed FWM at $500 \mathrm{~km}$ fiber range and $25 \mathrm{GHz}$ channel spacing, (b) With addressed FWM at $500 \mathrm{~km}$ fiber range and $25 \mathrm{GHz}$ channel spacing. 


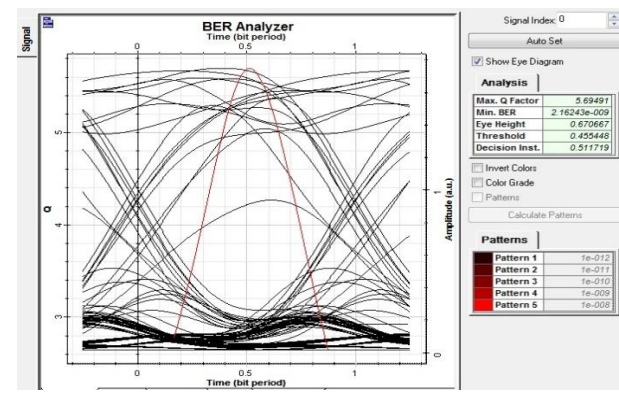

(a)

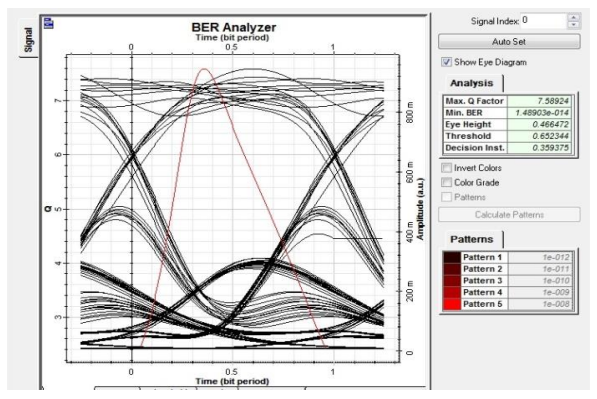

(b)

Figure 12. BER eye-diagrams. (a) Without addressed FWM at $500 \mathrm{~km}$ fiber range and $25 \mathrm{GHz}$ channel spacing, (b) With addressed FWM at $500 \mathrm{~km}$ fiber range and $25 \mathrm{GHz}$ channel spacing.

\section{Conclusions}

This work studies LHOCS that is the fundamental requirement of current communication network. The well known FWM is investigated and treated using IDSP receiver mechanism, using theoretical and simulation backgrounds. Moreover, the comparison between operating with and without managed FWM is explored for $500 \mathrm{~km}$ fiber span, -6 to $6 \mathrm{dBm}$ input power, receiver power, nonlinear effective area, nonlinear dispersion slope, channel spacing, and number of channels. The penalty of power by reason of FWM is also discussed and the improvement is quantified with IDSP receiver. Some extra unwanted effects such ASE and PMD noises are addressed using EDFA and Bessel filters. It is concluded from simulation analysis that $10^{-1}$ BER different is recorded between managed FWM and un-managed FWM. The good BER results show the effectiveness of the proposed LHOCS against FWM.

Author Contributions: Conceptualization, F.M., F.A.; methodology, F.M., F.A., G.A., Z.H.A.; software, F.M., M.B.; validation, F.M., G.A., M.B. ; formal analysis, F.M., F.A., G.A., S.H., M.B.; investigation, F.M., S.H., G.A., Z.H.A., M.B.; data analysis, F.M., F.A., G.A., Z.H.A., S.H., M.B.; writing-original draft, F.M., F.A.; writing-review and editing, G.A., Z.H.A., S.H., M.B., M.J.P., D.Y.S.; visualization, M.B., M.J.P., D.Y.S.; supervision, G.A., Z.H.A.; project administration, F.M., F.A., B.M.; funding acquisition, B.M., M.J.P., D.Y.S. All authors have read and agreed to the published version of the manuscript.

Funding: This work was supported This research was supported by Korea Electric Power Corporation. (Grant number: R18XA02).

Data Availability Statement: Not Applicable

Acknowledgments: This work was supported by the Ministry of Education and the Deanship of Scientific Research, Najran University. Kingdom of Saudi Arabia, under code number NU/ESCI/19/001.

Conflicts of Interest: The authors declare no conflict of interest.

\section{References}

1. Ali, F.; Khan, Y.; Qureshi, S.S.; Ahmad, S.; Waqas, M. Effect of fiberoptics nonlinearities in long-haul and ultra-high speed DWDM optical transmission networks at 10, 40 and $100 \mathrm{~Gb} / \mathrm{s}$ ultra-high speed data rates. J. Opt. Commun. 2018. 2018-0173. [CrossRef]

2. Xu, T.; Mikroulis, S.; Mitchell, J.E.; Darwazeh, I. Bandwidth compressed waveform for 60-GHz millimeter-wave radio over fiber experiment. J. Lightw. Technol. 2015, 34, 3458-3465. [CrossRef]

3. Ali, F.; Ahmad, S.; Muhammad, F.; Abbas, Z.H.; Habib, U.; Kim, S. Adaptive equalization for dispersion mitigation in multichannel optical communication networks. Electronics 2019, 8, 1364. [CrossRef]

4. $\quad \mathrm{Xu}$, T.; Shevchenko, N.A.; Lavery, D.; Semrau, D.; Liga, G.; Alvarado, A.; Killey, R.I.; Bayvel, P. Modulation format dependence of digital nonlinearity compensation performance in optical fibre communication systems. Opt. Express 2017, 25, 3311-3326. [CrossRef] [PubMed]

5. Piran, M.J.; Tran, N.H.; Suh, D.Y.; Song, J.B.; Hong, C.S.; Han, Z. QoE-Driven Channel Allocation and Handoff Management for Seamless Multimedia in Cognitive 5G Cellular Networks. IEEE Trans. Veh. Technol. 2017, 66, 6569-6585. [CrossRef]

6. Agrell, E.; Karlsson, M.; Chraplyvy, A.R.; Richardson, D.J.; Krummrich, P.M.; Winzer, P.; Roberts, K.; Fischer, J.K.; Savory, S.J.; Eggleton, B.J.; et al. Roadmap of optical communications. J. Opt. 2016, 18, 063002. [CrossRef] 
7. Cartledge, J.C.; Guiomar, F.P.; Kschischang, F.R.; Liga, G.; Yankov, M.P. Digital signal processing for fiber nonlinearities. Opt. Express 2017, 25, 1916-1936. [CrossRef] [PubMed]

8. Bakhshali, A.; Chan, W.Y.; Cartledge, J.C.; Sullivan, M.O.; Laperle, C.; Borowiec, A.; Roberts, K. Frequency-domain Volterra-based equalization structures for efficient mitigation of intrachannel Kerr nonlinearities. J. Light. Technol. 2016, 34, 1770-1777. [CrossRef]

9. Liu, X.; Chraplyvy, A.; Winzer, P.; Tkach, R.; Chandrasekhar, S.; Phase-conjugated twin waves for communication beyond the Kerr nonlinearity limit. Nat. Photonics 2013, 7, 560. [CrossRef]

10. Dong, Y.; Rawachy, E.A.; Giddings, R.P.; Jin, W.; Nesset, D.; Tang, J.M. Multiple Channel Interference Cancellation Of Digital Filter Multiple Access PONs. Int. J. Light. Technol. 2017, 35, 34-44. [CrossRef]

11. Piran, M.J.; Pham, S.M.R.Q.V.; Suh, D.Y.; Han, Z. Multimedia Communication over Cognitive Radio Networks from QoS/QoE Perspective: A Comprehensive Survey. J. Netw. Comput. Appl. 2020, 172, 1-44.

12. Hasegawa, T.; Yamamoto, Y.; Hirano, M. Optimal fiber design for large capacity long haul coherent transmission. Opt. Express 2017, 25, 706-712. [CrossRef]

13. Asif Khan, M.K.; Ali, F.; Irfan, M.; Muhammad, F.; Althobiani, F.; Ali, A.; Khan, S.; Rahman, S.; Perun, G.; Glowacz, A. Mitigation of Phase Noise and Nonlinearities for High Capacity Radio-over-Fiber Links. Electronics 2021, 10, 345. [CrossRef]

14. Kishikawa, H.; Uetai, M.; Gotoi, N. All-Optical Modulation Format Conversion Between OOK, QPSK, and 8QAM. J. Light. Technol. 2019, 37, 3925-3931. [CrossRef]

15. Liga, G.; Saavedra, G.; Bayvel, P. Combining Optical Phase Conjugation and Volterra Equalisation: A Novel Nonlinearity Compensation Scheme. J. Light. Technol. 2018, 36, 377-400.

16. Rademacher, G.; Luis, R.S.; Puttnam, B.J.; Maruyama, R.; Aikawa, K.; Awaji, Y.; Furukawa, H.; Petermann, K.; Wada, N. Investigation of Intermodal Nonlinear Signal Distortions in Few-Mode Fiber Transmission. J. Light. Technol. 2019, 37, 1-6. [CrossRef]

17. Kong, M.; Kong, M.; Li, X.; Zhang, J.; Xin, K.W.X.; Zhao, F.; Yu, J. High spectral efficiency 400 Gb/s transmission by different modulation formats and advanced DSP. J. Light. Technol. 2019. [CrossRef]

18. Al-Rawachy, E.; Giddings, R.P.; Tang, J. Experimental demonstration of a real-time digital filtermultiple access PON with low complexity DSP-based interference cancellation. J. Light. Technol. 2019, 37, 4315-4329. [CrossRef]

19. Giacoumidis, E.; Lin, Y.; Wei, J.; Aldaya, I.; Tsokanos, A.; Barry, L.P. Harnessing machine learning for fiber-induced nonlinearity mitigation in long-haul coherent optical OFDM. Future Internet 2018, 11, 2. [CrossRef]

20. Chen, Y.; Shen, S.; Zhou, Q.; Yao, S.; Zhang, R.; Omar, S. A reliable OFDM-based MMW mobile fronthaul with DSP-aided sub-band spreading and time-confined windowing. J. Light. Technol. 2019, 37, 3236-3243. [CrossRef]

21. Irfan, M.; Ali, F.; Muhammad, F.; Habib, U.; Alwadie, A.S.; Glowacz, A.; Abbas, Z.H.; Kaǹtoch, E. DSP-Assisted nonlinear impairments tolerant $100 \mathrm{Gbps}$ optical backhaul network for long-Haul transmission. Entropy 2020, 22, 1062. [CrossRef]

22. Anjum, O.F.; Bottrill, K.; Horak, P.; Jung, Y.; Suzuki, M.; Yamamoto, Y.; Hasegawa, T.; Richardson, D.J.; Parmigiani, F.; Petropoulos, P.; et al. Channel selective wavelength conversion by means of inter modal four wave mixing. In Proceedings of the Optical Fiber Communications Conference and Exhibition, San Diego, CA, USA, 3-7 March 2019; pp. 1-3.

23. Stojanovic, N.; Changsong, X. An efficient method for skew estimation and compensation in coherent receivers. IEEE Photonics Technol. Lett. 2016, 28, 489-492. [CrossRef]

24. Benyahya, K.; Simonneau, C.; Ghazisaeidi, A.; Barr, N.; Jian, P.; Morizur, J.F.; Labroille, G.; Bigot, M.; Sillard, P.; Provost, J.G.; et al. Multiterabit transmission over OM2 multimode fiber with wavelength and mode group multiplexing and direct detection. J. Light. Technol. 2018, 36, 355-360. [CrossRef]

25. Maharana, D.; Rout, R. A 4 channel WDM-based hybrid optical Fiber/FSO communication system using DP QPSK modulation for bit rate of 100/112 Gb/s. Int. J. Eng. Res. Technol. 2019, 8, 442-445.

26. Charlet, G.; Pecci, P. Ultra-long haul submarine transmission. In Undersea Fiber Communication Systems, 2nd ed.; Academic Press: San Diego, CA, USA, 2016; pp. 165-235.

27. Hui, R.; O'Sullivan, M. Optical system performance measurements. In Fiber Optic Measurement Techniques; Elsevier Academic Press: San Diego, CA, USA, 2009.

28. Zhuge, Q.; Chen, X. Advances in modulation and DSP for optical transmission systems. J. Opt. Commun. 2018, 409, 1-136. [CrossRef]

29. Okamoto, K. Planar lightwave circuits. In Fundamentals of Optical Waveguides, 2nd ed.; Elsevier Academic Press: San Diego, CA, USA, 2006; pp. 417-534.

30. Obaid, H.M.; Shahid, H. Achieving high gain using Er-Yb codoped waveguide/fiber optical parametric hybrid amplifier for dense wavelength division multiplexed system. Opt. Eng. 2018, 57, 056108. [CrossRef]

31. Niaz, A.; Qamar, F.; Islam, K.; Shahzad, A.; Shahzadi, R.; Ali, M. Performance analysis and comparison of QPSK and DP-QPSK based optical fiber communication systems. ITEE J. 2018, 7, 34-39.

32. JPaza, L.; Alvaradoc, Y.J.; Lascanoa, L.; Vera, C.C. Three levels of propagation of the four-wave mixing signal. J. Results Phys. 2019, 11, 414-421.

33. El-Naha, F.I. Coherent quadrature phase shift keying optical communication systems. Optoelectron. Lett. 2018, 14, 372-375. [CrossRef]

34. Kahn, J.M.; Miller, D.A.B. Communications expands its space. Nat. Photonics 2017, 11, 5-8. [CrossRef] 
35. Perin, J.K.; Shastri, A.; Kahn, J. Design of low-power DSP-free coherent receivers for data center links. J. Light. Technol. 2017, 35, 4650-4662. [CrossRef]

36. Miao, X.; Bi, M.; Fu, Y.; Li, L.; Hu, W. Experimental study of NRZ, Duobinary, and PAM-4 in O-band DML-based 100G-EPON. IEEE Photonics Technol. Lett. 2017, 29, 1490-1493. [CrossRef]

37. Irfan, M.; Ali, F.; Muhammad, F.; Alwadie, A.S.; Glowacz, A.; Goldasz, I.; Mielnik, R.; Alkahtani, F.S.; Khan, H. An Optimal Framework for WDM Systems Using Analytical Characterization of Refractive Index-Related Nonlinear Impairments. Electronics 2021, 10, 221. [CrossRef]

38. Khalid, R.; Zafrullah, M. Analysis of the Q-factor of the external modulation with different optical filters to overcome the fwm non-linearity in the fiber networks. In Proceedings of the International Symposium on Wireless Systems and Networks, Lahore, Pakistan, 19-22 November 2017; pp. 19-22.

39. Sahin, E.; Ooi, K.J.A.; Choi, J.W.; Ng, D.K.T.; Png, C.E.; Tan, D.H. Efficient four-wave mixing using CMOS-compatible ultrasiliconrich nitride photonic crystal waveguides.In Proceedings of the Conference on Lasers and Electro-Optics Pacific Rim, Hong Kong, China, 29 July-3 August 2018; pp. 1-2.

40. Panda, A.; Mishra, D.P. Nonlinear effect of four wave mixing for wdm in radio-over-fiber systems. J. Electron. Commun. Eng. Res. 2014, 2, 1-6.

41. Solanki, P.B.; Al-Rubaiai, M.; Tan, X. Extended Kalman Filter-Based Active Alignment Control for LED Optical Communication. IEEE/ASME Trans. Mechatron. 2018, 23, 1501-1511. [CrossRef]

42. Muhammad, F.; Ali, F.; Habib, U.; Usman, M.; Khan, I.; Kim, S. Time domain equalization and digital back-propagation method-based receiver for fiber optic communication systems. Int. J. Opt. 2020, 2020, 3146374. [CrossRef]

43. Jiangbing, D.; Li, L.; Xinyu, F.; Qingwen, L.; Zuyuan, H. Sensitivity enhancement for fiber bragg grating sensors by four wave mixing. Photonics 2015, 2, 426-443.

44. Anjum, O.F.; Horak, P.; Jung, Y.; Suzuki, M.; Yamamoto, Y.; Hasegawa, T.; Petropoulos, P.; Richardson, D.J.; Parmigiani1, F. Bandwidth enhancement of inter-modal our wave mixing Bragg scattering by means of dispersion engineering. APL Photonics 2018, 4, 368-370.

45. Ali, F.; Khan, Y.; Qureshi, S.S. Transmission performance comparison of $16^{*} 100 \mathrm{Gbps}$ dense wavelength division multiplexed long haul optical networks at different advance modulation formats under the influence of nonlinear impairments. J. Opt. Commun. 2019. [CrossRef]

46. Marvin, S.; Pratheesh, P. Analysis of SPM and FWM in optical fiber communication system using optisystem. Int. J. Eng. Res. Technol. 2014, 3, 1700-1704.

47. Ali, F.; Muhammad, F.; Habib, U.; Khan, Y.; Usman, M. Modeling and minimization of FWM effects in DWDM-based long-haul optical communication systems. Photonic Netw. Commun. 2020. [CrossRef]

48. Ali, F.; Khan, Y.; Ali, A.; Ahmad, G. Minimization of nonlinear impairments and its impact on transmission performances of highcapacity long-haul optical networks. J. Opt. Commun. 2018. [CrossRef]

49. Rademacher, G.; Ruben, S.; Benjamin, J.; Furukawa, H.; Maruyama, R. Investigation of intermodal four-wave mixing for nonlinear signal processing in few-mode fibers. IEEE Photonics Technol. Lett. 2018, 30, 1527-1530. [CrossRef]

50. Chen, Z.; Guo, X.; Fu, X.; Shu, C.; Li, Z. Investigation of four-wave mixing crosstalk in phase-sensitive fiber optical parametric amplifier. J. Light. Technol. 2018, 36, 5113-5120. [CrossRef]

51. Zhang, H.; Jin, L.; Zhang, H.; Xu, Y.; Shi, L.; Wang, T.; Chen, H.; Wang, D.; Ma, X. All-fiber nonlinear optical switch based on polarization controller coiled SMF-GIMF-SMF for ultrashort pulse generation. J. Opt. Commun. 2019, 452, 7-11. [CrossRef] 